\title{
Mobile technology habits: patterns of association among device usage, intertemporal preference, impulse control, and reward sensitivity
}

\author{
Henry H. Wilmer ${ }^{1}$ Jason M. Chein ${ }^{1}$
}

Published online: 15 March 2016

(C) Psychonomic Society, Inc. 2016

\begin{abstract}
Mobile electronic devices are playing an increasingly pervasive role in our daily activities. Yet, there has been very little empirical research investigating how mobile technology habits might relate to individual differences in cognition and affect. The research presented in this paper provides evidence that heavier investment in mobile devices is correlated with a relatively weaker tendency to delay gratification (as measured by a delay discounting task) and a greater inclination toward impulsive behavior (i.e., weaker impulse control, assessed behaviorally and through self-report) but is not related to individual differences in sensitivity to reward. Analyses further demonstrated that individual variation in impulse control mediates the relationship between mobile technology usage and delay of gratification. Although based on correlational results, these findings lend some backing to concerns that increased use of portable electronic devices could have negative impacts on impulse control and the ability to appropriately valuate delayed rewards.
\end{abstract}

Keywords Cognitive and attentional control · Impulse control $\cdot$ Reward sensitivity $\cdot$ Technology

Electronic devices have become more and more portable and convenient, providing nearly constant (and ever more efficient) access to the Internet and a diverse range of software applications and digital media. With this ease of access, technology is playing an increasingly large role in our mental

Jason M. Chein

jchein@temple.edu

1 Department of Psychology, Temple University, 1701 N. 13th Street, Philadelphia, PA 19122, USA lives, serving as a form of "extended cognition" (Barr, Pennycook, Stolz, \& Fugelsang, 2015; Clark \& Chalmers, 2002; Clayton, Leshner, \& Almond, 2015). This situation can be viewed as a double-edged sword: although it allows us to communicate, learn, and entertain ourselves, it also makes it difficult to avoid doing so-even when engaging with technology is likely to detract from other ongoing activities. Notifications built into smartphones and other e-devices can intrude on three of our five senses, with lights, tones, and vibrations each beckoning us to extricate ourselves from our current tasks and engage instead with the device. Even in the absence of notifications, internal and external cues (a thought about work or a social relationship, something brushing against your pocket, noticing others on their phones, etc.) provide regular reminders of the opportunity to engage with the digital world.

These constant notifications and cues, and the relative immediacy with which we can acquire information and satisfy specific desires by responding to them, may alter our basic cognitive and affective functioning. Regular intrusions into ongoing cognition present a challenge to the self-regulatory, cognitive control processes that support the maintenance of goal-directed behaviors. And, by offering an often-gratifying escape from ongoing tasks, engagement with e-devices may occupy basic reward-related processes and even impact the fundamental mechanisms through which we valuate and process rewards (Atchley \& Warden, 2012). Indeed, some have argued that today's youth - referred to at times in the popular media as the "Now Generation" and "Generation C" (for "Connected"; "Introducing Generation C," 2012), having grown up in an era in which mobile technology is omnipresent, possess an especially strong need for instant gratification, which has diminished their ability to plan effectively for the future (Muther, 2013). Such assertions are part of a larger movement generally espousing the ills of technology access 
and use (Bauerlein, 2008; Ellison, 2012; Greenfield, 2013; Sutter, 2012). Unfortunately, most of the relevant assertions (e.g., today's youth are more immediacy oriented) are based principally on anecdote, while empirical evidence regarding any relationship between technology habits and delay of gratification (or other aspects of cognition) is still quite limited. Some foundational work, such as that of Atchley and Warden (2012), shows a close parallel between the willingness to delay the receipt of monetary rewards and to delay responding to informational prompts (to text or call someone back). These findings indicate that technology behaviors can be understood in terms of frequently researched decision-making processes (i.e., intertemporal preference), though the specific mechanisms that are most directly linked to regular technology use remain poorly understood.

Prior research on intertemporal preference (Kalenscher \& Pennartz, 2008; Peters \& Büchel, 2011; van den Bos \& McClure, 2013) has established that individual differences in the inclination to forego a smaller near-term reward in favor of a larger delayed reward (i.e., to delay gratification) relates to the behavior of two interacting systems: one governing the capacity to control impulses and the other influencing the individual's sensitivity to immediately available rewards (McClure, Laibson, Loewenstein, \& Cohen, 2004). Put differently, the tendency to seek immediate gratification can be explained either by weak impulse control (i.e., the inability to withhold a reactive or reflexive response in favor of more deliberative actions; Ainslie, 1975) or greater immediate reward sensitivity (i.e., the tendency to seek out novel or rewarding sensations and to experience greater sensation upon acquiring a reward; Carver \& White, 1994).

As the opportunities for technology use have grown, so too has a body of literature investigating the resultant cognitive and behavioral impacts (cf. Baumgartner, Weeda, van der Heijden, \& Huizinga, 2014; Minear, Brasher, McCurdy, Lewis, \& Younggren, 2013; Ralph, Thomson, Seli, Carriere, \& Smilek, 2014; Wang \& Tchernev, 2012). Understandably, a significant area of focus in recent research is the safety implications of using a cellphone while driving (e.g. Atchley, Atwood, \& Boulton, 2011; Strayer \& Drews, 2007). For example, work in this field has demonstrated that individuals who have a tendency to text on their cellphones while driving show a steeper discounting function compared to those who do not (Hayashi, Russo, \& Wirth, 2015). That is, those who more frequently engage in this dangerous behavior are also generally less inclined to delay gratification in favor of a larger, later reward. This work shows that at least one technology-related habit - texting while driving — is related to variation in intertemporal preference. Whether this relationship arises from individual differences in impulse control or reward sensitivity (or some other correlated variable), and whether it generalizes to other mobile technology habits, remains undetermined.
Additional clues come from work by Pearson, Murphy, and Doane (2013) and Sanbonmatsu, Strayer, Medeiros-Ward, and Watson (2013). As in the aforementioned studies, Pearson and colleagues examined cell-phone use while driving, and explored possible relationships with individual traits that are related to impulse control and reward sensitivity (using the Urgency Premeditation Perseverance Sensation Seeking Impulsive Behavior Scale [UPPS]; Whiteside \& Lynam, 2001). Likewise, Sanbonmatsu et al. (2013) asked participants to report how often they used their cellphones while driving, assessed a broader facet of technology engagement captured by the Media Multitasking Index (Ophir, Nass, \& Wagner, 2009), and also examined trait impulsivity (Barratt Impulsiveness Scale, Version 11; Patton, Stanford, \& Barratt, 1995) and sensation seeking (Sensation Seeking Scale; Zuckerman, Eysenck, \& Eysenck, 1978). In both studies, a significant relationship was found between the assessed technology habits and the individual trait questionnaires.

These findings encourage the conclusion that personality variables related to both impulsivity and reward processing are relevant factors in mobile technology use. Still, some concern has been raised about the specificity, utility, and reliability of the particular questionnaires used in those studies (Gray \& Wilson, 2007; Zuckerman, 2007). For instance, as noted by Zuckerman (1996; see also Zuckerman, 2007) the original Sensation Seeking Scale contains a number of "anachronistic" questions and may be too narrowly focused on specific contextualized activities; limitations also adopted (in revised form) into the UPPS. Zuckerman and colleagues have since constructed a revised scale meant to better capture overall sensation seeking (Zuckerman, Kuhlman, Joireman, Teta, \& Kraft, 1993). Subsequently, Steinberg et al. (2008) introduced the use of only a carefully selected subset of questions from the revised scale to disentangle impulsivity and reward/ sensation seeking.

In our pursuit of the question of what motivates smartphone usage, we hoped to delineate in a single study the interrelationships between smartphone usage, delay of gratification, impulse control, and reward sensitivity. In so doing, we first sought to develop a survey instrument with a focus on smartphone usage. Researchers have already developed a large number of self-report indices of technology use (Alloway \& Alloway, 2012; Jacobsen \& Forste, 2011; Junco, 2012; Ophir et al., 2009), but there exists little consensus on which instruments most aptly capture relevant individual differences. One recent, and already widely deployed, measure of technology usage is the aforementioned Media Multitasking Index. While media multitasking is certainly an important aspect of technology use, this instrument focuses on only this facet of technology-related behavior (multitasking with technology) and does not isolate the type of usage that differentiates smartphones from other technology (short, frequent usage throughout the day). Thus, we sought to develop 
an ecumenical, but still targeted, assessment of personal mobile technology device usage.

With this assessment, we sought to determine if individuals who reported heavier mobile technology use also exhibit a differential tendency to delay gratification, as measured by performance on a "delay discounting" task. Merely establishing a relationship between technology habits and delay of gratification would not be sufficient to clarify the factors that drive this relationship, whether differences in impulse control or differences in reward processing. So, we further assessed individual differences in both impulse control and reward sensitivity to determine which, if either, of these variables mediates the relationship between technology engagement and delay of gratification. To avoid the pitfalls of relying on individual, and potentially conflated, questionnaire instruments, we followed Steinberg et al. (2008) in using a validated subset of questions from existing questionnaires (as explained in the Method section), and additionally collected responses from secondary measures of both impulse control and reward sensitivity in order to develop construct-level estimates of each variable.

\section{Method}

\section{Participants}

Participants were 91 undergraduate students $(71.4 \%$ female; age $M=20.05, S D=2.19$ ) who completed a battery of questionnaires and cognitive tests. The sample was racially $(59.3 \%$ self-identified as Caucasian or white, $15.4 \%$ as African American or black, $14.3 \%$ as Asian, $1.1 \%$ as American Indian or Alaskan Native, $3.3 \%$ as more than one race, and $6.6 \%$ declined to respond) and ethnically (4.4\% self-identified as Hispanic) diverse. All procedures were approved by the Institutional Review Board at Temple University, and participants were given course credit for participation.

\section{Measures}

Technology engagement We created a technology engagement scale with the purpose of indexing mobile technology usage. ${ }^{1}$ By assessing self-reported behaviors regarding different facets of mobile technology, we hoped to create an index that could characterize individual technology engagement patterns while not being overly biased by any one technologyrelated habit. The three components of this scale were brief self-report questionnaires assessing (1) phone-based social

\footnotetext{
${ }^{1}$ We also assayed other technology-related habits (e.g., computer use, video gaming), but responses to these questions were not considered in the present study.
}

media use, (2) frequency of public status updating, and (3) phone-checking behavior. Phone-based social media use was determined by the participants' responses to three Likert-style questions about their daily usage of various mobile social media applications (Facebook, Twitter, Instagram, Vine, Snapchat). Frequency of posting public status updates was determined by the participants' response to a single question, "How often do you post public status updates?" Phonechecking behavior was determined by the average response of participants' answers to three Likert-style questions: "How often do you check your phone for new activity?", "How often do you find yourself checking your phone when you have a few moments to spare?", and "How often do you find yourself checking your phone during conversations or when hanging around with friends?" The study sample indicated acceptable internal reliability for this construct $(\alpha=.65)$. To further explore the validity of this technology engagement scale, we also gathered, from a subset of our participants, information regarding their technology multitasking habits using the Media Multitasking Index (MMI; Ophir et al., 2009) and examined correlations between the Technology Engagement scale and the MMI. The MMI provides an estimate of the amount of time one spends multitasking with various forms of media. In the standard form, participants are asked to estimate the total number of hours they spend engaging in 12 different forms of media (e.g., watching television, playing video games, talking on the phone, instant messaging) and to specify, across a series of pages (one for each media type), the degree to which they use each media technology concurrently with each of the other media formats (i.e., engage in media multitasking). The MMI score is an aggregated score based on the sum total of multitasking habits (specific calculation is described in Ophir et al., 2009). For expediency, in the present study we created a matrix-style version of the MMI (see supplemental online information), which allowed participants to detail their media multitasking habits on a single computerized form rather than across a series of repeated forms pertaining to each media type.

Intertemporal preference We assessed individual differences in the tendency to delay gratification in favor of larger, later, rewards using a delay discounting task (O'Brien, Albert, Chein, \& Steinberg, 2011). In the delay discounting task, participants were asked to make hypothetical choices between a smaller sum of money offered now versus a larger sum of money (always $\$ 1,000$ ) offered at six different delays, ranging from 1 day to 1 year. The smaller sum of money offered was varied systematically, until the participant reached an indifference point - the value at which the subjective value of the smaller immediate offer matched the subjective value of the larger $(\$ 1,000)$ delayed offer (Ohmura, Takahashi, Kitamura, \& Wehr, 2006). Participants completed 10 trials at each delay interval. Using this data, we calculated each individual's 
discount rate $(k)$ as well as their indifference points at each delay. As is commonly done, a natural log transformation was applied to all $k$ values in order to reduce skewness to an acceptable level. While we investigated indifference points at each delay, based on previous experience with this task (O'Brien et al., 2011; Weigard, Chein, Albert, Smith, \& Steinberg, 2014) we expected the responses to the longer delays to have the greatest individual subject variance. Thus, the longest two delays ( 6 months and 1 year) were averaged and taken as a more sensitive index of individual variation in immediacy orientation.

Reward sensitivity Two instruments were used to create a reward sensitivity construct: a subset of questions from Zuckerman's revised Impulsive Sensation Seeking scale and a subscale of the BIS/BAS questionnaire. The Impulsive Sensation Seeking measure (Zuckerman et al., 1993) is a 19item self-report questionnaire that intentionally conflates impulsive and sensation-seeking behaviors in order to broadly characterize these personality traits. To isolate sensation seeking, Steinberg et al. (2008) identified a subset of six items from the updated Zuckerman scale that most purely related to this construct ("I like to have new and exciting experiences and sensations, even if they are a little frightening," "I like doing things just for the thrill of it," "I sometimes like to do things that are a little frightening," "I'll try anything once," "I sometimes do 'crazy' things just for fun," and "I like wild and uninhibited parties"). These items were answered as either true (coded 1 ) or false (coded 0 ), and item scores were averaged to create a mean Sensation Seeking score. This subset of items has been shown to exhibit good internal consistency ( $\alpha=.70$; Steinberg et al., 2008). In the current sample, the internal consistency was similarly good $(\alpha=.73)$.

The BIS/BAS scales are measures of behavioral inhibition and behavioral approach (Carver \& White, 1994). For the purposes of the present study, we were primarily concerned with the behavioral approach component (BAS), which is itself comprised three subscales: Fun Seeking, Reward Responsiveness, and Drive. Because we were specifically focused on targeting individual reward sensitivity, only the Reward Responsiveness subscale was used in our analyses. This subscale has been shown to have good internal consistency $(\alpha=.73$; Carver $\&$ White, 1994). The present sample indicated acceptable internal consistency $(\alpha=.68)$ with this subscale.

Impulse control An Impulse Control construct was calculated by taking the average score from two measures, Barratt Impulsiveness Scale and false alarm rate on a go/no-go task. The Barratt Impulsiveness Scale is a widely used self-report measure of impulsivity (Patton et al., 1995). Again, based on the findings of Steinberg et al. (2008), we elected to use only 18 items of the full 30-item questionnaire having specificity with respect to impulsive behavior (rather than to sensation seeking). Each item was answered on a 4-point scale (rarely/ never, occasionally, often, almost always) and scores were averaged, with higher scores indicative of greater impulsivity. Steinberg et al. (2008) showed that this subset of questions has good internal consistency $(\alpha=.73)$. In the current sample, the internal consistency was similarly good $(\alpha=.75)$.

The go/no-go task used in the current study involved the rapid presentation of a series of go $(x)$ and no-go $(k)$ stimuli. Participants were instructed to give a button press response following each $x$, but to withhold responding whenever they saw a $k$ stimulus. The stimuli were presented for $250 \mathrm{~ms}$ each, followed by an unpredictable ITI ranging between $750 \mathrm{~ms}$ and $1,750 \mathrm{~ms}$. In total, 333 stimuli were presented, of which only 50 were no-go trials $(k \mathrm{~s})$. These no-go trials were pseudorandomly interspersed into the series so that a no-go trial was equally often preceded by 1 to 10 prior go trials (five occurrences of each). The entire task lasted just over $8.5 \mathrm{mi}-$ nutes. Normalized scores from both the Barratt Impulsiveness Scale and go/no-go measures were inverted so as to reflect impulse control rather than impulsivity (i.e., a higher score on the construct indicated a stronger tendency to control impulsive responses).

\section{Results}

\section{Descriptive statistics and bivariate correlations}

Basic descriptive statistics for each measure are provided in Table 1, and correlations between individual measures are shown in Table 2. To verify the validity of our Technology Engagement scale, we first examined the bivariate correlation between normalized scores on this scale and the MMI scores obtained from a subset of our participants $(n=50)$. The significant correlation $(r=.310, p=.028)$ indicates that, despite focusing on different aspects of technology use, the two measures explain overlapping variance with respect to general technology habits.

\section{Relationship between technology engagement and intertemporal preference}

A primary aim of the present study was to determine whether there is a relationship between technology use and intertemporal preference. Indeed, we found a significant correlation between individuals' discounting rate $(\log k)$ and their self-reported technology engagement $(r=.240, p=.023)$. Next, we confirmed that the correlation was driven by participants' responses at the longest 2 delays. As expected, technology engagement scores were highly correlated with the mean indifference points for 6-month and 1-year delays ( $r=$ $-.286, p=.006$; see Fig. 1a), but not for any of the shorter 
Table 1 Descriptive statistics for individual measures

\begin{tabular}{|c|c|c|c|c|c|}
\hline & & Minimum & Maximum & Mean & Std. Deviation \\
\hline \multirow{4}{*}{ Technology engagement } & MMI & 0.01 & 7.31 & 3.22 & 1.61 \\
\hline & Phone-based social media use & 4 & 25 & 12.32 & 4.76 \\
\hline & Phone-checking behavior & -2.72 & 1.59 & -0.03 & 0.78 \\
\hline & Frequency of posting public status updates & 1 & 7 & 2.36 & 1.15 \\
\hline Intertemporal Preference (ITP) & Mean indifference point & 57.43 & 970.13 & 489.41 & 249.03 \\
\hline \multirow[t]{2}{*}{ Impulse control } & Go /No-Go false alarms & 2 & 44 & 19.95 & 8.88 \\
\hline & Barratt Impulsiveness Scale & 1.22 & 2.93 & 2.03 & 0.37 \\
\hline \multirow[t]{2}{*}{ Reward sensitivity } & Zuckerman's Impulsive Sensation Seeking scale & 0 & 1 & 0.68 & 0.29 \\
\hline & BAS-reward & 13 & 20 & 16.57 & 1.86 \\
\hline
\end{tabular}

delays (all $p \mathrm{~s}>.05$, for the average of indifference points at the shorter delays: $r=-.020, p=.849)$.

\section{Relationship between technology engagement, impulse control, and reward sensitivity}

We next sought to determine whether there was also a relationship between technology engagement and either impulse control or reward sensitivity. Bivariate correlations revealed a significant negative relationship between technology engagement and impulse control ( $r=-.234, p=.025$; see Fig. 1b), indicating that individuals who report more engagement with e-devices tend to exhibit a lack of impulse control. Meanwhile, no such relationship existed between technology engagement and reward sensitivity $(r=.052, p=.627$; see Fig. 1c).

\section{Mediation of the relationship between technology engagement and delay of gratification}

The pattern of correlations we obtained suggested the possibility that the relationship between technology engagement and intertemporal preference might be specifically mediated by individual variation in impulse control, and not in reward sensitivity. To test this possibility we conducted mediation analyses using the bootstrapping methods delineated by Preacher and Hayes (2004) and utilizing Hayes' PROCESS Model (Hayes, 2013; Preacher \& Hayes, 2004). Each analysis was performed using 10,000 bootstrap resamples to estimate the indirect effect of the proposed mediator variables. The bootstrapping method yields $95 \%$ confidence intervals (CIs) for each proposed mediator and its indirect effect. If zero is not included within an estimated $95 \% \mathrm{CI}$, the indirect effect is taken to be significantly different from zero. In an initial mediation analysis we tested whether impulse control plays a mediating role in the relationship between technology engagement and intertemporal preference. The indirect effect of technology engagement through impulse control yielded a bootstrapped CI that did not include zero $(b=.059,95 \% \mathrm{CI}$ $[0.005,0.187])$, indicating that impulse control is indeed a significant mediator of the relationship. That is, as shown in Fig. 2, while higher levels of technology engagement are related to a tendency toward accepting a smaller immediate reward, this relationship is due in part to the relationship between technology engagement and impulse control.

A further mediation analysis sought to determine whether reward sensitivity mediates the relationship between technology engagement and intertemporal preference. This mediation analysis yielded a bootstrapped CI that included zero, indicating that reward sensitivity is not a mediator in this relationship $(b=.012,95 \%$ CI $[-0.037,0.116])$.

\section{Discussion}

By combining different aspects of mobile technology use into a single scale, we were able to obtain a novel metric of individual differences in mobile technology usage. Importantly,

Table 2 Correlations between individual measures ${ }^{\wedge}=$ reverse-coded; $* p<.05 ; * * p<.01$

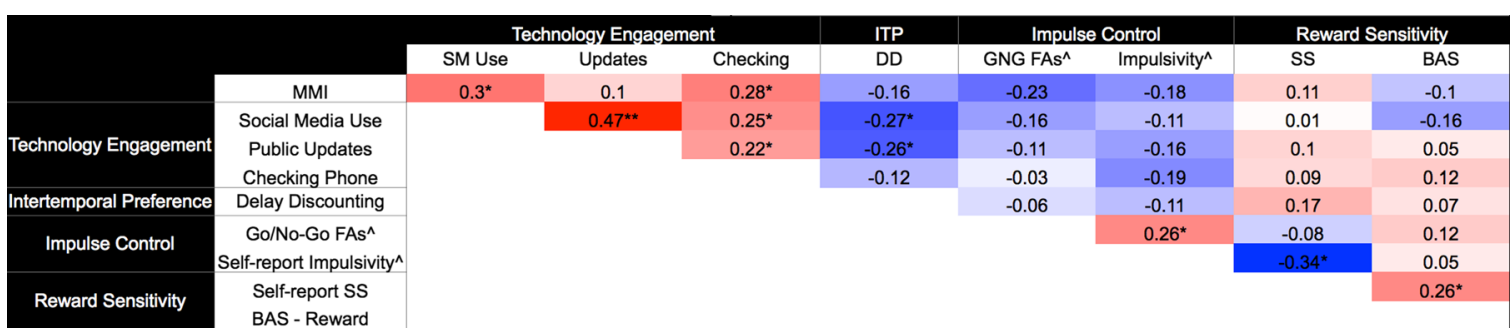




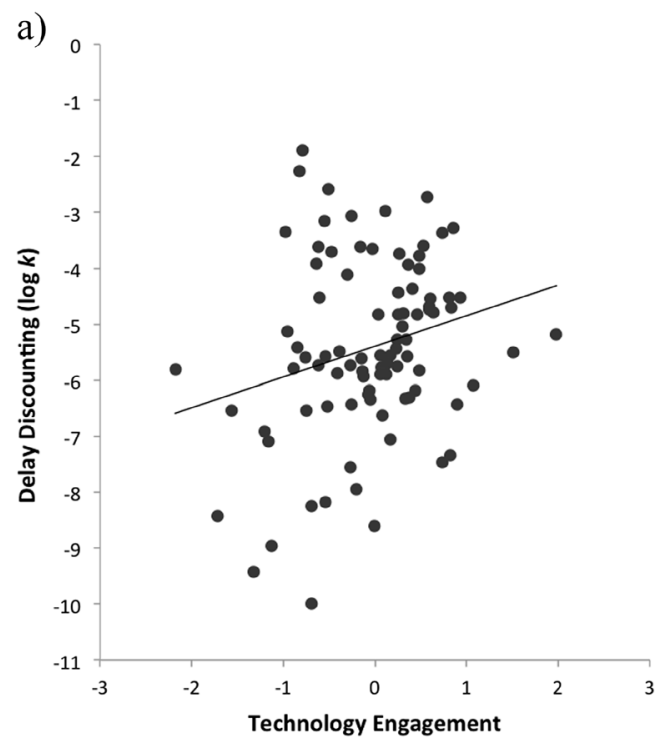

b)

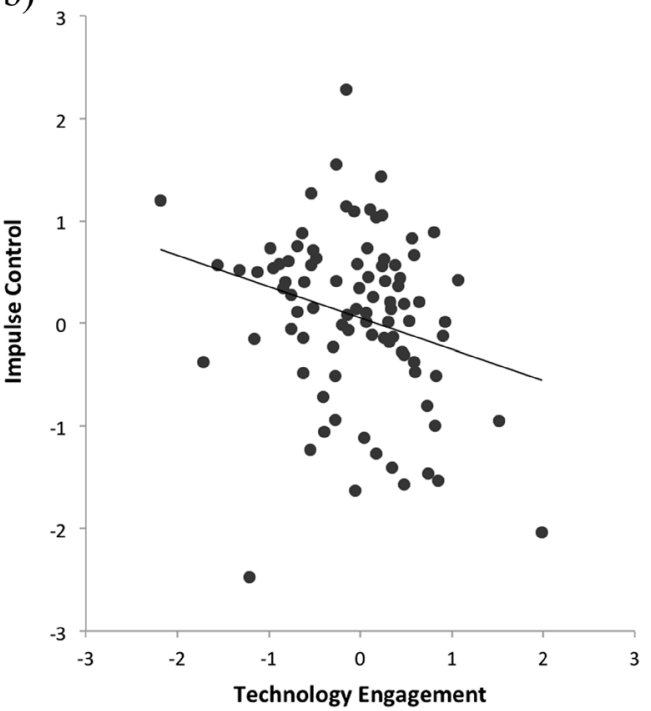

c)

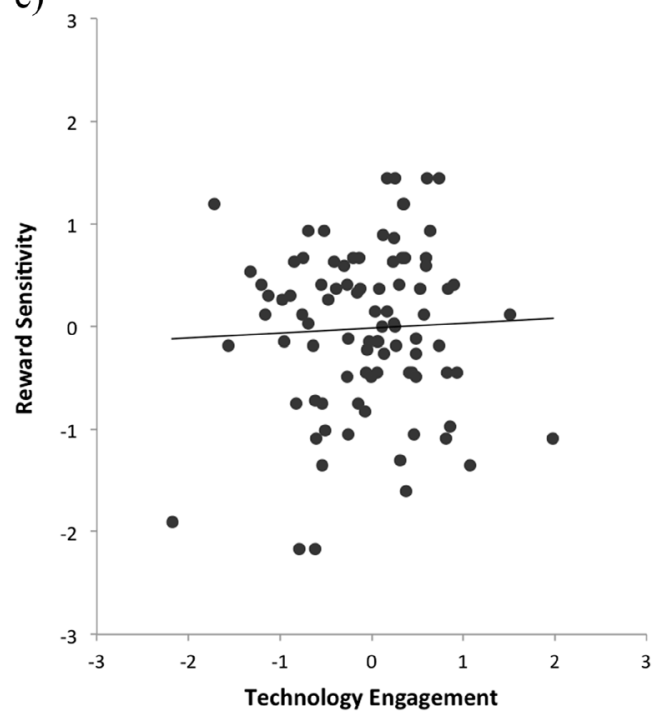

Fig. 1 Relationship between technology engagement and normalized (a) intertemporal preference $(\log k)$, (b) impulse control, and (c) reward sensitivity

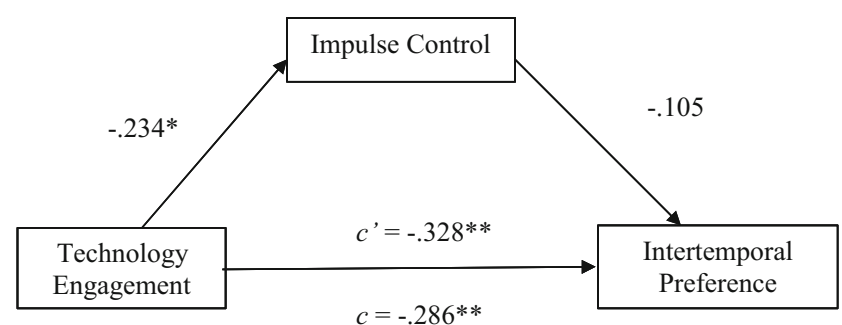

Fig. 2 Impulse Control mediates the relationship between technology engagement and intertemporal preference

this method of assessing technology habits proved to be positively correlated with another widely used index of technology habits (the MMI), indicating a common underlying behavior. Our Technology Engagement scale thus appears to be an effective measure that encapsulates various ways by which people engage with mobile technology, with a specific focus on the type of behaviors that are typical of smartphone and other portable e-device usage.

Interestingly, the results suggest that a significant relationship exists between technology engagement and intertemporal preference. Whereas Atchley and Warden (2012) demonstrated similarities in the processes by which information and monetary rewards are valuated, and Hayashi et al. (2015) linked delay tolerance with cellphone use while driving, the present research provides evidence that broader aspects of technology use are also related to individual differences in intertemporal preference.

It has been argued that performance on delay discounting tasks is dependent upon two different aspects of decision making: impulse control and reward processing. In order to pinpoint the source of the differential behavior exhibited by heavy technology users, we examined the relationship between technology engagement and both of these purported facets of decision making. Similar to a previously reported association between media multitasking and impulsivity (Minear et al., 2013), our analyses revealed that impulse control significantly correlated with technology engagement. Furthermore, impulse control was a significant mediator of the relationship between technology engagement and intertemporal preference, whereas reward sensitivity was not. Indeed, our results fail to indicate even a basic relationship between technology engagement and reward-driven behaviors (cf. Pearson et al., 2013; Sanbonmatsu et al., 2013). The apparent discrepancy between the earlier and current findings may relate to our efforts to carefully dissociate the impulse control and reward sensitivity constructs. However, we acknowledge that other factors, including an emphasis on disparate aspects of technology-related behavior, and overall differences in design and statistical power, may have contributed to the different results.

The current findings extend a growing literature demonstrating the links between technology habits and aspects of 
cognitive functioning-for example, working memory (Alloway \& Alloway, 2012), attention (Paul, Baker, \& Cochran, 2012), and executive functioning (Abramson et al., 2009) - and has potentially important implications for our understanding of the factors that motivate technology use habits. In considering the question "What drives people to engage with their smartphones?", one might offer two seemingly reasonable answers: (1) individuals are unable to withhold the impulse to check, whether driven by endogenous thoughts or exogenous cues, and (2) individuals engage with their phones in an attempt to seek out a rewarding stimulus. Ultimately, the present evidence leads us to the conclusion that mobile technology habits, such as frequent checking, are driven most strongly by uncontrolled impulses and not by the desire to pursue rewards.

Despite this intriguing pattern of results, several clear limitations should be acknowledged, with important implications for the conduct of future research in this arena. Most important, although we provide evidence of mediation, the data are correlational in nature. Thus, the true causal relationships could go in either direction: (1) individual differences in intertemporal preference and impulse control may influence the extent to which people engage with mobile technology, or (2) a prior history of investment in mobile technology may have a lasting effect on impulse control and intertemporal preference. Further research is needed to determine the directionality of the effect.

Another important limitation of this study is that the data we collected to form the Technology Engagement scale was generated from self-report. Accordingly, it is possible that the results reflect differences in how one subjectively perceives his or her own technology habits but fail to accurately characterize the individual's actual usage patterns. Indeed, recent empirical evidence substantiates this possibility (Andrews, Ellis, Shaw, \& Piwek, 2015). As an increasing number of our behaviors and social interactions occur in the digital world, the field of research psychology would greatly benefit from the development of applications that objectively trace smartphone usage. $^{2}$

Developing a better understanding of the impacts of smartphone and mobile technology usage on cognitive and affective outcomes is critical in understanding the potential problems of overusing this technology. Given the correlational nature of the present data, we should remain cautious before drawing any causal inferences. Still, the findings provide important insights regarding the individual difference factors that relate to technology engagement and are consistent with the common conception that frequent smartphone use goes hand in hand with impatience and impulsivity.

\footnotetext{
$\overline{2}$ There are already several applications that track usage, but none designed specifically with the concerns of research in mind.
}

Acknowledgments The authors would like to recognize Samantha Stella, Elizabeth Atterbury, and Mary Caulfield for their important contributions in gathering the data for this research.

\section{References}

Abramson, M. J., Benke, G. P., Dimitriadis, C., Inyang, I. O., Sim, M. R., Wolfe, R. S., \& Croft, R. J. (2009). Mobile telephone use is associated with changes in cognitive function in young adolescents. Bioelectromagnetics, 30(April), 678-686. doi:10.1002/bem.20534

Ainslie, G. (1975). Specious reward: A behavioral theory of impulsiveness and impulse control. Psychological Bulletin, 82(4), 463-496. doi: $10.1037 / \mathrm{h} 0076860$

Alloway, T. P., \& Alloway, R. G. (2012). The impact of engagement with social networking sites (SNSs) on cognitive skills. Computers in Human Behavior, 28(5), 1748-1754. doi:10.1016/j.chb.2012.04. 015

Andrews, S., Ellis, D. A., Shaw, H., \& Piwek, L. (2015). Beyond selfreport: Tools to compare estimated and real-world smartphone use. PLoS ONE, 10(10), e0139004. doi:10.1371/journal.pone.0139004

Atchley, P., Atwood, S., \& Boulton, A. (2011). The choice to text and drive in younger drivers: Behavior may shape attitude. Accident Analysis and Prevention, 43(1), 134-142. doi:10.1016/j.aap.2010. 08.003

Atchley, P., \& Warden, A. C. (2012). The need of young adults to text now: Using delay discounting to assess informational choice. Journal of Applied Research in Memory and Cognition, 1(4), 229234. doi:10.1016/j.jarmac.2012.09.001

Barr, N., Pennycook, G., Stolz, J. A., \& Fugelsang, J. A. (2015). The brain in your pocket: Evidence that Smartphones are used to supplant thinking. Computers in Human Behavior, 48, 473-480. doi:10. 1016/j.chb.2015.02.029

Bauerlein, M. (2008). The dumbest generation: How the digital age stupefies young Americans and jeopardizes our future (Or, don't trust anyone under 30). New York, NY: Jeremy P. Tarcher/Penguin.

Baumgartner, S. E., Weeda, W. D., van der Heijden, L. L., \& Huizinga, M. (2014). The relationship between media multitasking and executive function in early adolescents. The Journal of Early Adolescence. doi:10.1177/0272431614523133

Carver, C. S., \& White, T. L. (1994). Behavioral inhibition, behavioral activation, and affective responses to impending reward and punishment: The BIS/BAS scales. Journal of Personality and Social Psychology, 67, 319-333. doi:10.1037/0022-3514.67.2.319

Clark, A., \& Chalmers, D. J. (2002). The extended mind (Reprinted). The Philosopher's Annual, XXI(1), 59-74. doi:10.1111/1467-8284. 00096

Clayton, R. B., Leshner, G., \& Almond, A. (2015). The extended iSelf: The impact of iPhone separation on cognition, emotion, and physiology. Journal of Computer-Mediated Communication, 1-17. doi: $10.1111 /$ jcc 4.12109

Ellison, K. (2012). Are smartphones making us dumber? Forbes. Retrieved from http://www.forbes.com/sites/netapp/2012/09/12/isan-digital-data-overload-shortening-our-attentions-spans-andmaking-us-dumber/

Gray, J. M., \& Wilson, M. A. (2007). A detailed analysis of the reliability and validity of the sensation seeking scale in a UK sample. Personality and Individual Differences, 42(4), 641-651. doi:10. 1016/j.paid.2006.08.019

Greenfield, S. (2013). Screen technologies [Video file]. Retrieved from http://www.susangreenfield.com/science/screen-technologies/

Hayashi, Y., Russo, C. T., \& Wirth, O. (2015). Texting while driving as impulsive choice: A behavioral economic analysis. Accident 
Analysis \& Prevention, 83, 182-189. doi:10.1016/j.aap.2015.07. 025

Hayes, A. F. (2013). Introduction to mediation, moderation, and conditional process analysis: A regression-based approach. New York, NY: The Guilford Press. doi:10.1111/jedm.12050

Introducing generation $\mathrm{C}$ : Americans 18-34 are the most connected. (2012, February 23). Nielsen Newswire. Retrieved from http:// www2.acnielsen.com/us/en/insights/news/2012/introducinggeneration-c.html

Jacobsen, W. C., \& Forste, R. (2011). The wired generation: Academic and social outcomes of electronic media use among university students. Cyberpsychology, Behavior and Social Networking, 14(5), 275-280. doi:10.1089/cyber.2010.0135

Junco, R. (2012). The relationship between frequency of Facebook use, participation in Facebook activities, and student engagement. Computers and Education, 58(1), 162-171. doi:10.1016/j. compedu.2011.08.004

Kalenscher, T., \& Pennartz, C. M. A. (2008). Is a bird in the hand worth two in the future? The neuroeconomics of intertemporal decisionmaking. Progress in Neurobiology, 84(3), 284-315. doi:10.1016/j. pneurobio.2007.11.004

McClure, S. M., Laibson, D. I., Loewenstein, G., \& Cohen, J. D. (2004). Separate neural systems value immediate and delayed monetary rewards. Science, 306(5695), 503-507. doi:10.1126/science. 1100907

Minear, M., Brasher, F., McCurdy, M., Lewis, J., \& Younggren, A. (2013). Working memory, fluid intelligence, and impulsiveness in heavy media multitaskers. Psychonomic Bulletin \& Review, 20, 1274-1281. doi:10.3758/s13423-013-0456-6

Muther, C. (2013, February 2). The growing culture of impatience, where instant gratification makes us crave more instant gratification. The Boston Globe. Retrieved from https://www.bostonglobe.com/ lifestyle/style/2013/02/01/the-growing-culture-impatience-whereinstant-gratification-makes-crave-more-instant-gratification/ q8tWDNGeJB2mm45fQxtTQP/story.html

O’Brien, L., Albert, D., Chein, J., \& Steinberg, L. (2011). Adolescents prefer more immediate rewards when in the presence of their peers. Journal of Research on Adolescence, 21(4), 747-753. doi:10.1111/j. 1532-7795.2011.00738.x

Ohmura, Y., Takahashi, T., Kitamura, N., \& Wehr, P. (2006). Threemonth stability of delay and probability discounting measures. Experimental and Clinical Psychopharmacology, 14(3), 318-328. doi:10.1037/1064-1297.14.3.318

Ophir, E., Nass, C., \& Wagner, A. D. (2009). Cognitive control in media multitaskers. Proceedings of the National Academy of Sciences of the United States of America, 106(37), 15583-15587. doi:10.1073/ pnas.0903620106

Patton, J. H., Stanford, M. S., \& Barratt, E. S. (1995). Factor structure of the Barratt Impulsiveness Scale. Journal of Clinical Psychology, 51(6), 768-774. doi:10.1002/1097-4679(199511)51:6<768

Paul, J. A., Baker, H. M., \& Cochran, J. D. (2012). Effect of online social networking on student academic performance. Computers in Human Behavior, 28(6), 2117-2127. doi:10.1016/j.chb.2012.06. 016

Pearson, M. R., Murphy, E. M., \& Doane, A. N. (2013). Impulsivity-like traits and risky driving behaviors among college students. Accident Analysis \& Prevention, 53, 142-148. doi:10.1016/j.aap.2013.01. 009
Peters, J., \& Büchel, C. (2011). The neural mechanisms of inter-temporal decision-making: Understanding variability. Trends in Cognitive Sciences, 15(5), 227-239. doi:10.1016/j.tics.2011.03.002

Preacher, K. J., \& Hayes, A. F. (2004). SPSS and SAS procedures for estimating indirect effects in simple mediation models. Behavior Research Methods, Instruments, \& Computers: A Journal of the Psychonomic Society, 36(4), 717-731. Retrieved from http://www. ncbi.nlm.nih.gov/pubmed/15641418

Ralph, B. C. W., Thomson, D. R., Seli, P., Carriere, J. S. A., \& Smilek, D. (2014). Media multitasking and behavioral measures of sustained attention. Attention, Perception, \& Psychophysics, 77(2), 390-401. doi:10.3758/s13414-014-0771-7

Sanbonmatsu, D. M., Strayer, D. L., Medeiros-Ward, N., \& Watson, J. M. (2013). Who multi-tasks and why? Multi-tasking ability, perceived multi-tasking ability, impulsivity, and sensation seeking. PLOS ONE, 8(1). doi:10.1371/journal.pone.0054402

Steinberg, L., Albert, D., Cauffman, E., Banich, M., Graham, S., \& Woolard, J. (2008). Age differences in sensation seeking and impulsivity as indexed by behavior and self-report: Evidence for a dual systems model. Developmental Psychology, 44(6), 1764-1778. doi: 10.1037/a0012955

Strayer, D. L., \& Drews, F. A. (2007). Cell-phone-induced driver distraction. Current Directions in Psychological Science, 16(3), 128-131. doi:10.1111/j.1467-8721.2007.00489.x

Sutter, J. D. (2012). On second thought: Maybe smartphones make us "superstupid"? CNN. Retrieved from http://www.cnn.com/2012/09/ 11/tech/mobile/smartphones-superstupid/

van den Bos, W., \& McClure, S. M. (2013). Towards a general model of temporal discounting. Journal of the Experimental Analysis of Behavior, 99(1), 58-73. doi:10.1002/jeab.6

Wang, Z., \& Tchernev, J. M. (2012). The "myth" of media multitasking: Reciprocal dynamics of media multitasking, personal needs, and gratifications. Journal of Communication, 62, 493-513. doi:10. $1111 / j .1460-2466.2012 .01641 . x$

Weigard, A., Chein, J., Albert, D., Smith, A., \& Steinberg, L. (2014). Effects of anonymous peer observation on adolescents' preference for immediate rewards. Developmental Science, 17, 71-78. doi:10. 1111/desc. 12099

Whiteside, S. P., \& Lynam, D. R. (2001). The five factor model and impulsivity: Using a structural model of personality to understand impulsivity. Personality and Individual Differences, 30(4), 669689. doi:10.1016/S0191-8869(00)00064-7

Zuckerman, M. (1996). Item revisions in the Sensation Seeking Scale Form V (SSS-V). Personality and Individual Differences, 20(4), 515. doi:10.1016/0191-8869(95)00195-6

Zuckerman, M. (2007). The Sensation Seeking Scale V (SSS-V): Still reliable and valid. Personality and Individual Differences, 43(5), 1303-1305. doi:10.1016/j.paid.2007.03.021

Zuckerman, M., Eysenck, S., \& Eysenck, H. J. (1978). Sensation seeking in England and America: Cross-cultural, age, and sex comparisons. Journal of Consulting and Clinical Psychology, 46(1), 139-149. doi:10.1037/0022-006X.46.1.139

Zuckerman, M., Kuhlman, D. M., Joireman, J., Teta, P., \& Kraft, M. (1993). A comparison of three structural models for personality: The Big Three, the Big Five, and the Alternative Five. Journal of Personality and Social Psychology, 65(4), 757-768. doi:10.1037/ 0022-3514.65.4.757 